\title{
A No-reference IQA Metric Based on BEMD and Riesz Tansform
}

\author{
Guangyi Yang \\ Wuhan University \\ School of Electronic Information \\ Wuhan, Hubei 430072, China \\ ygy@whu.edu.cn \\ Ye Zhi \\ Wuhan University \\ School of Electronic Information \\ Wuhan, Hubei 430072, China \\ yeyeye@whu.edu.cn
}

\author{
Yue Liao \\ Wuhan University \\ School of Electronic Information \\ Wuhan, Hubei 430072, China \\ liaoyue@whu.edu.cn \\ Jiahua Cao \\ Wuhan University \\ School of Electronic Information \\ Wuhan, Hubei 430072, China \\ jasoncao@whu.edu.cn
}

\author{
Qingyi Zhang \\ Wuhan University \\ School of Electronic Information \\ Wuhan, Hubei 430072, China \\ zhqy@whu.edu.cn \\ Deshi Li* \\ Wuhan University \\ School of Electronic Information \\ Wuhan, Hubei 430072, China \\ dsli@whu.edu.cn
}

\begin{abstract}
In recent years, the research of the no-reference (NR) image quality assessment (IQA) has been very active. But the NR-IQA research for noise-distorted images hasn't been proposed yet. In this paper, we propose a NR-IQA metric for noise-distorted images based on bidimensional empirical mode decomposition (BEMD) and Riesz transform, namely BRIQ index. Firstly, the denoised image is obtained by using the BEMD, Riesz transform and the visual contrast sensitivity function (CSF) to denoise the distorted image. Then the similarity calculation is performed using five Riesz transform feature maps of the distorted image and the denoised image. Finally, we combine these five similarity indices to obtain the final quality score for the distorted image. Experimental results on pubic database show that the BRIQ index evaluates the noise-distorted images with good performance, as consistent as subjective quality ratings.
\end{abstract}

\section{CCS CONCEPTS}

-Human-centered computing $\rightarrow$ Visualization design and evaluation methods;

\section{KEYWORDS}

no-reference, image quality assessment, bidimensional empirical mode decomposition, Riesz transform, human visual system

Permission to make digital or hard copies of all or part of this work for personal or classroom use is granted without fee provided that copies are not made or distributed for profit or commercial advantage and that copies bear this notice and the full citation on the first page. To copy otherwise, to republish, to post on servers or to redistribute to lists, requires prior specific permission and/or a fee.

MOBIMEDIA 2017, July 13-14, Chongqing, People's Republic of China Copyright @ 2017 EAI 978-1-63190-156-0
Conference on Mobile Multimedia Communications, Chongqing, China, July 2017 (MOBIMEDIA'2017), 5 pages.

DOI: $10.475 / 123 \_4$

\section{INTRODUCTION}

With the booming development of digital technology and multimedia communication, image quality assessment (IQA) research has raised a lot of attention in the past 20 years[6]. IQA can be divided into subjective assessment and objective assessment. Considering that the subjective IQA is unadaptable to industrial practical applications [17], the objective IQA has become the main research content in this field. According to the presence or absence of the reference image, the IQA can be divided into full-reference (FR), reducedreference (RR), and no-reference (NR) assessment[18].

As it's hard to obtain reference image in practical application, the study of NR-IQA has been very active in recent years. The NR-IQA can usually be divided into application-specific assessment and general purpose assessment. Considering that the general purpose NR-IQA is more difficult, most of the current studies focus on the application-specific assessment. Common types of distortion include JPEG compression distortion, blur distortion, noise distortion, and so on. Noise is an important factor in image distortion, but no studies investigated into this area previously, this paper is devoted to the study of NR-IQA for noise-distorted images with the human visual system (HVS) taken into consideration.

Empirical mode decomposition (EMD) is a signal processing method for non-stationary signal analysis proposed by Norden E. Huang et al.[5]. It can clearly distinguish the intrinsic mode functions (IMFs) of overlapping complex data without pre-setting the basis function as a wavelet decomposition. Nunes et al.[11] extended the one-dimensional EMD method to bidimensional application and proposed the bidimensional empirical mode decomposition (BEMD). As the research of the local feature analysis for IMF by Riesz transform[1], the function of Riesz transform has become more and more important in image processing. Considering the effect of BEMD on noise deduction when combined with Riesz transform[3], we propose a NR-IQA metric for 
noise-distorted images. We use this method to get denoised images from distorted images, and then compute feature maps corresponding to distorted and denoised images. Five similarity indices are obtained after the similarity caculation of feature maps. Finally, similarity indices are weighted and summed to obtain the final IQA score.

This paper is organized as follows: Section 2 first introduces the structural block diagram of the proposed NR-IQA metric, then introduces the BEMD. A brief introduction on Riesz transform and visual contrast sensitivity function (CSF) will also be included in this section. Finally the calculation formula of the proposed BRIQ index based on BEMD and Riesz Transform is obtained. Section 3 contains experimental analysis. Section 4 concludes this paper.

\section{THE PROPOSED ALGORITHM}

In this paper, the block diagram of the proposed BRIQ index based on BEMD and Riesz transform is shown in Figure 1. The distorted image $f$ is firstly decomposed by BEMD to obtain four image components $I M F 1, I M F 2$, $I M F 3$, and RESIDUE. Then, Log-Gabor filter[10], firstorder and second-order Riesz transform[8] are used on each component, and five groups of feature maps (each group of feature maps contains 4 image components) are obtained. The four image components in one group are weighted by CSF to get the feature maps $R_{x}\{g\}, R_{y}\{g\}, R_{x x}\{g\}, R_{x y}\{g\}$, and $R_{y y}\{g\}$ of denoised image $g$, which can be denoted as $g_{1}, g_{2}, g_{3}, g_{4}$, and $g_{5}$. At the same time, we obtain the five feature maps $R_{x}\{f\}, R_{y}\{f\}, R_{x x}\{f\}, R_{x y}\{f\}$, and $R_{y y}\{f\}$ by using Log-Gabor filter, first-order and second-order Riesz transform on the distorted image $f$, which can be denoted as $f_{1}, f_{2}, f_{3}, f_{4}$ and $f_{5}$. We calculate similarity on feature maps to acquire five similarity maps and use the average method to obtain five similarity indices $S_{i}(i=1,2, \ldots, 5)$. The five similarity indices are weighted and summed to obtain the final IQA score.

\subsection{Bidimensional Empirical Mode Decomposition (BEMD)}

For bidimensional $M \times N$ image signal $f(x, y), x=1, \ldots, M$, $y=1, \ldots, N$, BEMD implementation process[12] is shown as follows:

(1) Use 8 neighborhood pixels to find the local extreme point (maximum and minimum) of the image surface.

(2) Use the cubic spline interpolation metric to obtain the upper envelope $\mu_{\max }(x, y)$ and the lower envelope $\mu_{\min }(x, y)$ of the image surface.

(3) Determine the mean of the upper and lower envelopes $m(x, y)$.

(4) Subtract the mean envelope $m(x, y)$ from the image $f(x, y)$ :

$$
h(x, y)=f(x, y)-m(x, y)
$$

(5) Determine whether $h(x, y)$ satisfies the IMF screening termination condition:

$$
S D=\sum_{x=0}^{M} \sum_{y=0}^{N} \frac{\left|h_{j}(x, y)-h_{j-1}(x, y)\right|^{2}}{h_{j-1}^{2}(x, y)}
$$

Here we set $S D=0.2$.

Repeat from step 1 to step 5 until the criterion is satisfied, so we can obtain the first layer of the bidimensional IMF $I M F 1 c_{1}(x, y)$. The first layer residue $r_{1}(x, y)$ is obtained by subtracting $I M F 1$ from the original image. The $I M F$ $c_{j}(x, y)(j=2, \ldots, K)$ and the $K$ layer residua $r_{K}(x, y)$ are obtained successively when repeating step 1 to step 5 on the residue.

The final BEMD result is:

$$
f(x, y)=\sum_{j=1}^{K} c_{j}(x, y)+r_{K}(x, y)
$$

As the fact that the feature scale parameter is the actual measured data, the components obtained by the above method have obvious physical meaning, which characterizes the fluctuation and the frequency range of the signal on a certain feature scale parameter[16]. The image is decomposed, in the scale from small to large, into a series of detailed information in the order of "fine to coarse" and the large-scale trend information[7].

\subsection{Riesz Transform}

For the image $f(x, y)$, the first order Riesz transform can be expressed as:

$$
f_{R}(x, y)=\left(\begin{array}{l}
f_{x}(x, y) \\
f_{y}(x, y)
\end{array}\right)=\left(\begin{array}{l}
R_{x} * f(x, y) \\
R_{y} * f(x, y)
\end{array}\right)=\left(\begin{array}{l}
R_{x}\{f\}(x, y) \\
R_{y}\{f\}(x, y)
\end{array}\right)
$$

where $\left(R_{x}, R_{y}\right)$ represents the Riesz nucleus in the spatial domain.

Using the first-order Riesz transform, we can extract the local linear features of the image, but we can not express the corner points and intersection points in the image[19]. In order to characterize the various low-level features existing in the image, it is necessary to use higher order Riesz transform. In this paper, we use the second-order Riesz transform to express more complex features:

$$
\left\{\begin{array}{l}
R_{x x}\{f\}(x, y)=R_{x}\left\{R_{x}\{f\}\right\}(x, y) \\
R_{x y}\{f\}(x, y)=R_{x}\left\{R_{y}\{f\}\right\}(x, y) \\
R_{y y}\{f\}(x, y)=R_{y}\left\{R_{y}\{f\}\right\}(x, y)
\end{array}\right.
$$

Feature maps $f_{i}(i=1,2, \ldots, 5)$ are obtained by Log-Gabor filter, first-order and second-order Riesz transform of the distorted image respectively. Feature maps $g_{i j}(i=1,2, \ldots, 5 ; j=$ $1,2,3,4)$ are obtained by using the same method on four image components IMF1,IMF2,IMF3 and RESIDUE.

\subsection{Visual Contrast Sensitivity Function (CSF)}

At present, physiology and psychology has established a variety of HVS models to simulate the significant features of visual perception. Since CSF can reflect the subjective visual 


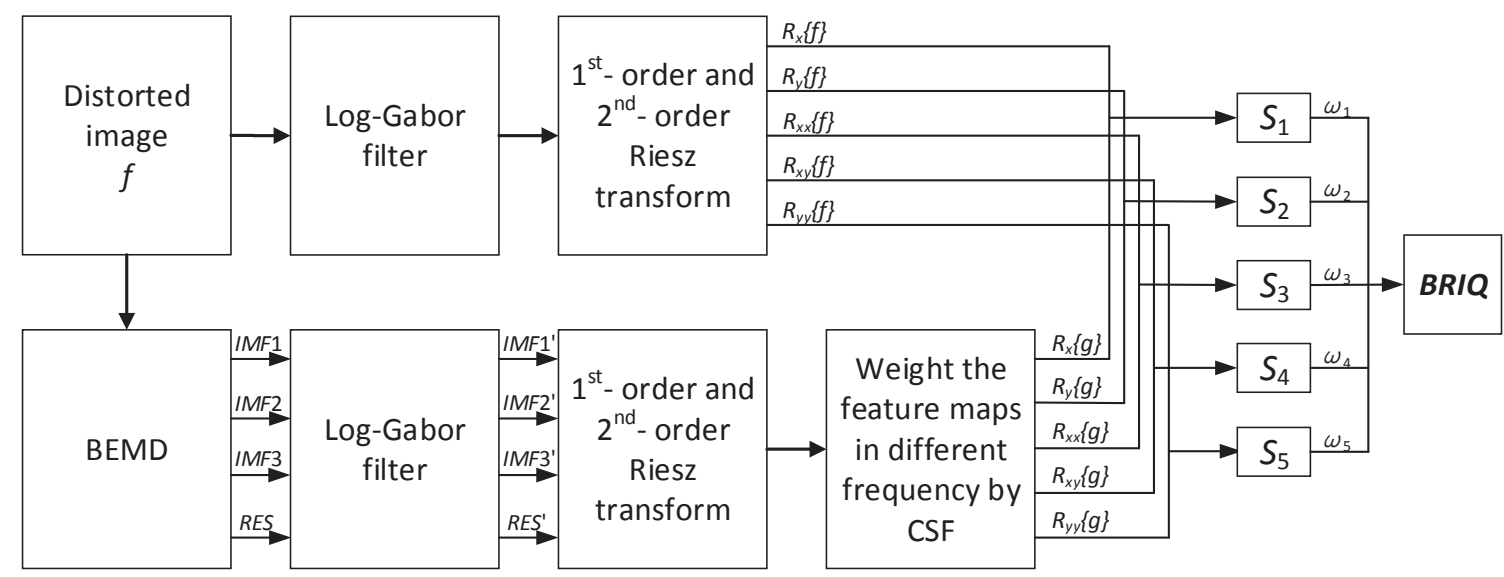

Figure 1: Illustration of the Proposed BRIQ Index.

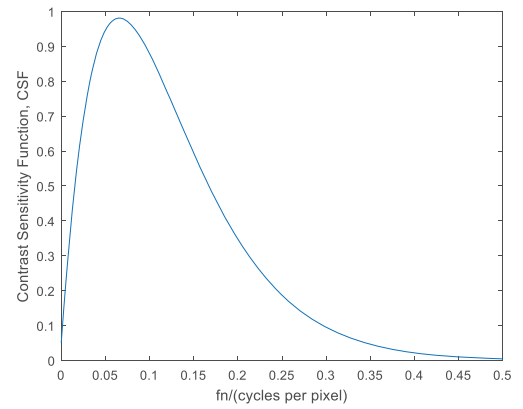

Figure 2: Curve of contrast sensitivity function.

experience, it has been applied to many IQA metrics [4]. In this paper, we use the CSF model proposed by Mannos et al. [9]:

$$
A\left(f_{r}\right) \approx 2.6\left(0.0192+0.114 f_{r}\right) \exp ^{-\left(0.114 f_{r}\right)^{1.1}}
$$

where $f_{r}$ is the spatial frequency, in units of cycle per degree. As the spatial frequency $f_{r}$ is the function of the image size and distance, we normalize it as $f_{n} \in[0,0.5]$. The feature curve of normalized CSF is shown in Fig. 2.

It can be seen from the CSF curve in Fig. 2 that the contrast sensitivity of the CSF curve is larger at the intermediate frequency region than that at the low frequency and high frequency regions. The decomposed distorted image by BEMD have four components: IMF1, IMF2, IMF3 and $R E S I D U E$, the main information of which are in different frequency. It is obvious that the contrast sensitivity of HVS among them is different too. In order to make the final assessment result more close to the subjective perception, we can use CSF to weight the different image components.

Assuming that the contrast sensitivity of corresponding image components, IMF1, IMF2,IMF3, and RESIDUE, are $C S F_{j}(j=1,2,3,4)$, respectively. Then:

$$
g_{i}=\sum_{j=1}^{4} g_{i j} \cdot C S F_{j}
$$

among them, $g_{i}(i=1,2, \ldots, 5)$ are five feature maps of the denoised image $g$. In this paper, $C S F_{j}(j=1,2,3,4)$ are determined as: $C S F_{1}=0.12, C S F_{2}=0.98, C S F_{3}=0.13$, $C S F_{4}=0.01$.

\subsection{BRIQ Index}

The similarity map calculation formula is defined as[18]:

$$
s_{i}(x, y)=\frac{2 f_{i}(x, y) g_{i}(x, y)+C_{i}}{f_{i}^{2}(x, y)+g_{i}{ }^{2}(x, y)+C_{i}}
$$

where $s_{i}(x, y)$ is the similarity map of $f_{i}$ and $g_{i}(i=1,2, \ldots, 5)$. $f_{i}(x, y)$ and $g_{i}(x, y)$ are the pixel values of the feature maps $f_{i}$ and $g_{i}$ at $(x, y)$, respectively. $C_{i}(i=1,2, \ldots, 5)$ are very small positive values to avoid the instability caused by the denominator being zero or close to zero. In this paper, we take $C_{i}=0.01(i=1,2, \ldots, 5)$.

After obtaining the five similarity maps, we use the following average formula to obtain five similarity indices:

$$
S_{i}=\frac{\sum_{x=1}^{M} \sum_{y=1}^{N} s_{i}(x, y)}{M \times N}
$$

among which $S_{i}(i=1,2, \ldots, 5)$ are taken as the similarity indices of feature maps $f_{i}$ and $g_{i}$.

Finally, we use $\operatorname{SROCC}_{i}(i=1,2, \ldots, 5)$ as the weight of the five similarity indices $S_{i}(i=1,2, \ldots, 5)$ which is obtained by fitting the similarity indices $S_{i}(i=1,2, \ldots, 5)$ of each feature map with DMOS respectively, and the final IQA score can be defined as:

$$
B R I Q=\frac{\sum_{i=1}^{5} S_{R O C C_{i}} \cdot S_{i}}{\sum_{i=1}^{5} S_{R O C C_{i}}}
$$

where $\operatorname{SROCC}_{i}(i=1,2,3,4,5)$ are determined as: $S R O C C_{1}=$ 0.9569, $S_{R O C C_{2}}=0.9530, S_{R O C} C_{3}=0.9686, S_{R O C C_{4}}=$ $0.9536, S_{R O C C_{5}}=0.9560$.

\section{EXPERIMENTAL ANALYSIS}

In order to verify the performance of BRIQ index, we ran tests on LIVE database[15]. The LIVE database contains 29 reference images and 779 distorted images, whose distortion 
Table 1: Performance Comparison of SROCC/KROCC/PLCC/RMSE on Live Database

\begin{tabular}{cccccc}
\hline \multirow{2}{*}{ Model } & \multirow{2}{*}{ FR/NR } & \multicolumn{4}{c}{ LIVE } \\
& & SROCC & KROCC & PLCC & RMSE \\
\hline PSNR & FR & 0.985 & 0.894 & 0.980 & 5.551 \\
SSIM & FR & 0.964 & 0.852 & 0.983 & 5.135 \\
FSIM & FR & 0.965 & 0.839 & 0.972 & 6.590 \\
FRIQUEE & NR & 0.982 & 0.886 & 0.898 & 12.287 \\
BLIINDSII & NR & 0.960 & 0.826 & 0.969 & 6.896 \\
BRIQ & NR & 0.967 & 0.847 & 0.976 & 6.059 \\
\hline
\end{tabular}

types include JPEG2000, JPEG compression, white noise, Gaussian blur and Rayleigh fading. The database provides the Differential Mean Opinion Scores (DMOS) for each image, and smaller DMOS value represents higher image quality.

In this paper, 5-parameter nonlinear logistic regression function is used to fit the data[14]. The performance of the metric is evaluated by four assessment indices[2]: Spearman rank-order correlation coefficient (SROCC), Kendall rank-order correlation coefficient (KROCC), Pearson linear correlation coefficient (PLCC), and root mean squared error (RMSE).

$$
f(x)=\beta_{1}\left[\frac{1}{2}-\frac{1}{1+\exp \left(\beta_{2}\left(x-\beta_{3}\right)\right)}\right]+\beta_{4} x+\beta_{5}
$$

where $x$ is the objective IQA score, $f(x)$ is the IQA regression fitting score, $\beta_{1}, \beta_{2}, \beta_{3}, \beta_{4}$ and $\beta_{5}$ are regressing function parameters.

Table 1 lists out the different IQA metrics for LIVE database. As can be seen from Table 1, BRIQ index has great performance on LIVE database.

Figure 3 shows the scatter plots of four different metric$\mathrm{s}$ for noise-distorted images in the LIVE database, where SSIM[18] and FSIM[20] are classical FR-IQA metrics, and BLIINDSII[13] and BRIQ proposed in this paper are NR-IQA metrics. It can be seen from Figure 3 that the scatter plot of the algorithm BRIQ is evenly distributed in the whole coordinate system and has a strong linear relationship with DMOS, which proves the robust performance of BRIQ index.

\section{CONCLUSIONS}

In this paper, an effective NR-IQA metric for noise-distorted images named BRIQ index is proposed by combining BEMD and Riesz transform with appropriate application of CSF curve. The metric is tested on LIVE database, and the scatter plots experiment is carried out on LIVE database. The experimental results show that the BRIQ index has a good performance in evaluating the noise-distorted images.

\section{REFERENCES}

[1] Paula Cerejeiras and Uwe Kähler. 2015. Monogenic signal theory. Operator theory (2015), 1701-1724.

[2] P Corriveau and A Webster. 2003. Final report from the video quality experts group on the validation of objective models of video quality assessment, phase II. Tech. Rep. (2003).

[3] Patrick Flandrin, Gabriel Rilling, and Paulo Goncalves. 2004 Empirical mode decomposition as a filter bank. IEEE signal processing letters 11, 2 (2004), 112-114.

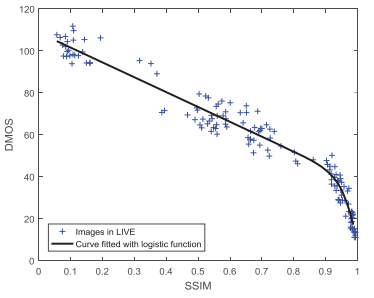

(a)

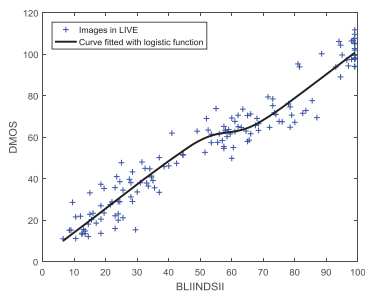

(c)

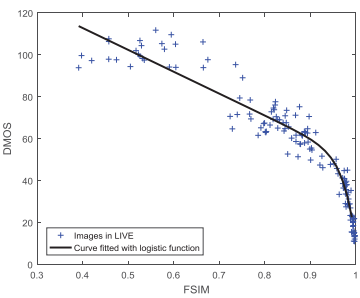

(b)

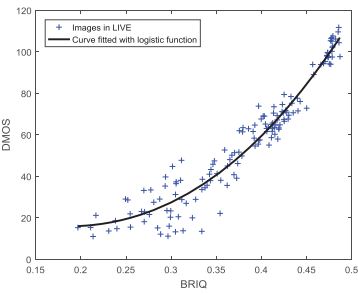

(d)
Figure 3: Scatter plots of predicted quality scores on LIVE database. From left to right, top to bottom are respectively the (a) SSIM, (b) FSIM, (c) BLIINDSII and (d) BRIQ.

[4] Xinbo Gao, Wen Lu, Dacheng Tao, and Xuelong Li. 2010. Image quality assessment and human visual system. In Visual Communications and Image Processing 2010. International Society for Optics and Photonics, 77440Z-77440Z.

[5] Norden E Huang, Zheng Shen, Steven R Long, Manli C Wu, Hsing H Shih, Quanan Zheng, Nai-Chyuan Yen, Chi Chao Tung, and Henry H Liu. 1998. The empirical mode decomposition and the Hilbert spectrum for nonlinear and non-stationary time series analysis. In Proceedings of the Royal Society of London A: Mathematical, Physical and Engineering Sciences, Vol. 454. The Royal Society, 903-995.

[6] Weisi Lin and C-C Jay Kuo. 2011. Perceptual visual quality metrics: A survey. Journal of Visual Communication and Image Representation 22, 4 (2011), 297-312.

[7] Steven R Long. 2005. Applications of HHT in image analysis. Hilbert-Huang Transform and Its Applications (2005), 289-305.

[8] Xue-Gang Luo, Hua-Jun Wang, and Sen Wang. 2015. Monogenic signal theory based feature similarity index for image quality assessment. AEU-International Journal of Electronics and Communications 69, 1 (2015), 75-81.

[9] James Mannos and David Sakrison. 1974. The effects of a visual fidelity criterion of the encoding of images. IEEE transactions on Information Theory 20, 4 (1974), 525-536.

[10] Javier R Movellan. 2002. Tutorial on Gabor filters. Open Source Document (2002).

[11] Jean Claude Nunes, Yasmina Bouaoune, Eric Delechelle, Oumar Niang, and $\mathrm{Ph}$ Bunel. 2003. Image analysis by bidimensional empirical mode decomposition. Image and vision computing 21 , 12 (2003), 1019-1026.

[12] Jean Claude Nunes, Oumar Niang, Yasmina Bouaoune, Eric Delechelle, and $\mathrm{Ph}$ Bunel. 2003. Bidimensional empirical mode decomposition modified for texture analysis. In Scandinavian Conference on Image Analysis. Springer, 171-177.

[13] Michele A Saad, Alan C Bovik, and Christophe Charrier. 2012. Blind image quality assessment: A natural scene statistics approach in the DCT domain. IEEE Transactions on Image Processing 21, 8 (2012), 3339-3352.

[14] Hamid R Sheikh, Muhammad F Sabir, and Alan C Bovik. 2006. A statistical evaluation of recent full reference image quality assessment algorithms. IEEE Transactions on image processing 15, 11 (2006), 3440-3451.

[15] Hamid R Sheikh, Zhou Wang, Lawrence Cormack, and Alan C Bovik. 2005. LIVE image quality assessment database release 2. 

(2005).

[16] Jian Wan, Longtao Ren, and Chunhui Zhao. 2008. Image feature extraction based on the two-dimensional empirical mode decomposition. In Image and Signal Processing, 2008. CISP'08. Congress on, Vol. 1. IEEE, 627-631.

[17] Zhou Wang, Alan C Bovik, and Ligang Lu. 2002. Why is image quality assessment so difficult?. In Acoustics, Speech, and Signal Processing (ICASSP), 2002 IEEE International Conference on, Vol. 4. IEEE, IV-3313.

[18] Zhou Wang, Alan Conrad Bovik, Hamid Rahim Sheikh, and Eero P Simoncelli. 2004. Image quality assessment: from error visibility to structural similarity. IEEE Transactions on Image Processing 13, 4 (2004), 600-612.

[19] Lin Zhang, Lei Zhang, and Xuanqin Mou. 2010. RFSIM: A feature based image quality assessment metric using Riesz transforms. In Image Processing (ICIP), 2010 17th IEEE International Conference on. IEEE, 321-324.

20] Lin Zhang, Lei Zhang, Xuanqin Mou, and David Zhang. 2011. FSIM: a feature similarity index for image quality assessment. IEEE transactions on Image Processing 20, 8 (2011), 2378-2386. 